\title{
Influence of MLH1 on colon cancer sensitivity to poly(ADP-ribose) polymerase inhibitor combined with irinotecan
}

\author{
LUCIO TENTORI $^{1 *}$, CARLO LEONETTI $^{4 *}$, ALESSIA MUZI $^{1}$, ANNALISA SUSANNA DORIO $^{1}$, \\ MANUELA PORRU ${ }^{4}$, SUSANNA DOLCI $^{2}$, FEDERICA CAMPOLO ${ }^{2}$, PATRIZIA VERNOLE ${ }^{3}$, \\ PEDRO MIGUEL LACAL ${ }^{5}$, FRANÇOISE PRAZ ${ }^{6,7}$ and GRAZIA GRAZIANI ${ }^{1}$
}

\begin{abstract}
Departments of ${ }^{1}$ System Medicine, ${ }^{2}$ Biomedicine and ${ }^{3}$ Science and Technology of Education, University of Rome 'Tor Vergata', I-00133 Rome; ${ }^{4}$ Experimental Preclinical Laboratory, Regina Elena National Cancer Institute, I-00158 Rome; ${ }^{5}$ Laboratory of Molecular Oncology, Istituto Dermopatico Dell'Immacolata (IDI), Istituto di Recovero e Cura a Carattere Scientifico (IRCCS)', I-00167 Rome, Italy; ${ }^{6}$ INSERM, UMR_S 938, Saint-Antoine Research Centre, F-75012 Paris; ${ }^{7}$ UPMC Univ Paris 06, UMR_S 938, Saint-Antoine Research Centre, F-75012 Paris, France
\end{abstract}

Received January 16, 2013; Accepted March 1, 2013

DOI: $10.3892 /$ ijo.2013.1932

\begin{abstract}
Poly(ADP-ribose) polymerase inhibitors (PARPi) are currently evaluated in clinical trials in combination with topoisomerase I (Top1) inhibitors against a variety of cancers, including colon carcinoma. Since the mismatch repair component MLH1 is defective in $10-15 \%$ of colorectal cancers we have investigated whether MLH1 affects response to the Top1 inhibitor irinotecan, alone or in combination with PARPi. To this end, the colon cancer cell lines HCT116, carrying MLH1 mutations on chromosome 3 and HCT116 in which the wildtype MLH1 gene was replaced via chromosomal transfer $(\mathrm{HCT} 116+3)$ or by transfection of the corresponding MLH1 cDNA (HCT116 1-2) were used. HCT116 cells or HCT116+3 cells stably silenced for PARP-1 expression were also analysed. The results of in vitro and in vivo experiments indicated that MLH1, together with low levels of Top1, contributed to colon cancer resistance to irinotecan. In the MLH1-proficient cells SN-38, the active metabolite of irinotecan, induced lower levels of DNA damage than in MLH1-deficient cells, as shown by the weaker induction of $\gamma-\mathrm{H} 2 \mathrm{AX}$ and p53 phosphorylation. The presence of MLH1 contributed to induce of prompt Chk1 phosphorylation, restoring G2/M cell cycle checkpoint and repair of DNA damage. On the contrary, in the absence of MLH1, HCT116 cells showed minor Chk1 phosphorylation and underwent apoptosis. Remarkably, inhibition of PARP
\end{abstract}

Correspondence to: Dr Grazia Graziani, Department of System Medicine, University of Rome 'Tor Vergata', Via Montpellier 1, I-00133 Rome, Italy

E-mail: graziani@uniroma2.it

*Contributed equally

Key words: colon cancer, chemotherapy, drug resistance, DNA repair, poly(ADP-ribose) polymerase, mismatch repair function by PARPi or by PARP-1 gene silencing always increased the antitumor activity of irinotecan, even in the presence of low PARP-1 expression.

\section{Introduction}

Colorectal cancer (CRC) is the second most common cause of cancer-related death in many industrialized countries. Although the addition of oxaliplatin or of the topoisomerase I (Top1) inhibitor irinotecan to 5-fluorouracil (5-FU)/ leucovorin regimen has greatly improved survival (1), treatment failure frequently occurs. Resistance to Top1 poisons has been generally attributed to overexpression of efflux pumps and low expression or mutations of Top1 (2). Strategies to counteract resistance to Top1 poisons include their combination with poly(ADP-ribose) polymerase (PARP) inhibitors (PARPi) (3-7). PARP-1 is the oldest member of a family of enzymes that synthesizes and transfers ADP-ribose polymers to acceptor proteins (including PARP-1 itself) using NAD ${ }^{+}$ as a substrate. PARP-1 has a key role in the surveillance and maintenance of genome integrity, sensing DNA breaks and signalling damage to DNA repair pathways (8). The interaction of poly(ADP-ribosyl)ated PARP-1 with specific domains of Top1 results in disjoining of Top1-DNA cleavable complex and favours resealing of strand breaks by the ligase activity of Top1 (9). Thus, PARPi would remove the antagonistic effect exerted by poly(ADP-ribose) on the mechanism of action of Top1 poisons, contributing to the formation of persistent DNA breaks (10). Another explanation for the synergism between PARPi and irinotecan implies the intervention of the base excision repair (BER) in the repair of the DNA damage induced by the Topl poison (5).

The MMR that eliminates replication errors and maintains genomic stability can be defective in familial or sporadic CRC and contributes to drug resistance/sensitivity (11). MMR is composed of several proteins forming different heterodimers: MutS $\alpha$ (MSH2/MSH6) recognizes single base mismatches, as 
well as 1-bp insertion-deletion loops, whereas MutS $\beta$ (MSH2/ MSH3) primarily recognizes 2-4-bp insertion-deletion loops; MutL $\alpha$, MLH1/PMS2), forms a ternary complex with a MutS heterodimer that binds to mismatches during replication, recruiting other proteins to complete the repair process (11). MutS $\alpha$ and MutL $\alpha$ also contribute to signal transduction pathways which lead to growth arrest or cell death induced by the methylating agent temozolomide (TMZ) $(12,13)$.

While the influence of MutS $\alpha$ and MutL $\alpha$ function in TMZ-induced sensitivity is known, the role of MLH1 in CRC susceptibility to irinotecan is controversial $(6,14-19)$. On the other hand, MLH1-deficiency confers resistance to cisplatin or carboplatin, but not to oxaliplatin (20).

Aims of the present study were to clarify whether MLH1 loss of function affects colon cancer sensitivity to irinotecan and whether lack of PARP-1 expression/activity has different outcome depending on MLH1 status.

\section{Materials and methods}

Cell lines and transfection. The colon cancer HCT116 cell line has a hemizygous nonsense mutation in the MLH1 gene located on chromosome 3 (21). The MLH1-proficient HCT116/3-6 cell line $(\mathrm{HCT} 116+3)$ was created by microcell chromosome transfer of a single normal human chromosome (22) and kindly provided by Dr Giancarlo Marra (Institute of Molecular Cancer Research, University of Zürich, Switzerland). The HCT116 1-2 and HCT116 0-1 cells were generated by transfection with the full-length wild-type MLH1 cDNA cloned into the pcDNA3.1/Hygro vector or with the empty control vector, respectively (15). Cell lines were cultured in DMEM (SigmaAldrich, Milan, Italy), supplemented with $10 \%$ fetal calf serum, $2 \mathrm{mM}$ L-glutamine and antibiotics. The HCT116+3 cells were cultured in the presence of $400 \mu \mathrm{g} / \mathrm{ml}$ geneticin (Sigma-Aldrich), the HCT116 1-2 and HCT116 0-1 cells in the presence of $100 \mu \mathrm{g} / \mathrm{ml}$ hygromycin (Sigma-Aldrich).

Stable silencing of PARP-1 in HCT116+3 cells (HCT116+3 $\mathrm{SiP})$ was obtained by co-transfection of the pBS-U6-SiP912 vector and the pBabe vector, carrying the puromycin resistance gene. HCT116+3 cells, transfected with pBabe vector only, were used as control (HCT116+3 Babe). The HCT116 SiP and Babe cell lines were previously described (23). Cells were maintained in the presence of $1 \mu \mathrm{g} / \mathrm{ml}$ puromycin (SigmaAldrich).

Drugs for in vitro studies. SN-38 (2.5 mM, Alexis), TMZ (100 mM, Sigma-Aldrich) and 7-hydroxystaurosporine (UCN-01, 100 nM, Sigma-Aldrich) stock solutions were prepared by dissolving the drugs in dimethyl sulfoxide. The final concentration of dimethyl sulfoxide was always $<0.5 \%$ (v/v) and did not contribute to toxicity. The PARP inhibitor GPI 15427 [10-(4-methyl-piperazin-1-ylmethyl)-2H-7-oxa-1,2diazabenzo[de]anthracen-3-one, Eisai, Baltimore, MD, USA] stock solution ( $1 \mathrm{mM}$ ) was prepared by dissolving GPI 15427 in $70 \mathrm{mM}$ PBS without potassium (24).

Cell growth assays. Cell proliferation of colon cancer cell lines was evaluated by colony formation assay. Cells were seeded in triplicate into 6 -well plates $\left(2 \times 10^{2} /\right.$ well $)$ and, after overnight incubation, treated with SN-38, TMZ or GPI 15427. Cells were cultured to allow colony-formation; after 10 days colonies were fixed, stained with $2 \%$ methylene blue in $95 \%$ ethanol and counted. Only colonies comprising $>50$ cells were scored as survival colonies. Chemosensitivity was evaluated in terms of $\mathrm{IC}_{50}$, i.e., the concentration of the drug capable of inhibiting cell growth by $50 \%$.

Flow cytometry analysis. Apoptosis and cell cycle perturbations induced by the drugs were evaluated by flow cytometry analysis of the DNA content. Untreated or drug treated cells $\left(1 \times 10^{6}\right)$ were washed with PBS and fixed in $70 \%$ ethanol at $-20^{\circ} \mathrm{C}$ for $\geq 18 \mathrm{~h}$. Cells were then centrifuged, suspended in $1 \mathrm{ml}$ of a solution containing $0.1 \%$ (w/v) sodium citrate, $0.1 \%$ Triton-X (v/v), $50 \mu \mathrm{g} / \mathrm{ml}$ propidium iodide, $10 \mu \mathrm{g} / \mathrm{ml}$ RNase and incubated in the dark at $37^{\circ} \mathrm{C}$ for $15 \mathrm{~min}$. The fluorescence was measured on a linear scale using a FACScan flow cytometer and the CellQuest software. Data collection was gated using forward light scatter and side scatter to exclude cell debris and aggregates. Apoptotic cells were represented by a broad hypodiploid peak easily distinguishable from the diploid DNA content in the red fluorescence channel. For cell cycle analysis, the Mod-Fit software version 3.0 was used (Becton-Dickinson, San Jose, CA, USA).

Western blot analysis. For immunoblot analysis the following primary antibodies were used: rabbit polyclonal anti-human phosphorylated p53 (Ser15) (Cell Signaling Technology; Beverly, MA, USA; 1:1,000 dilution); rabbit polyclonal anti-human phosphorylated Chk2 (Thr68) (Cell Signaling Technology; 1:1,000); rabbit polyclonal anti-human $\beta$-tubulin (clone H-235; Santa Cruz Biotechnology Inc., Santa Cruz, CA, USA; 1:400); rabbit polyclonal anti-human $\gamma$-H2AX (phosphoS139) (Abcam, Cambridge, UK; 1:1,000); mouse monoclonal antibody anti-human Chk1 (G4, Santa Cruz Biotechnology Inc; 1:500); rabbit monoclonal anti-human phosphorylated Chk1 (Ser345) (133D3, Cell Signaling Technology; 1:1,000); mouse monoclonal anti-human MLH1 (clone G168-15, BD Biosciences, San Jose, CA, USA; 1/500); mouse monoclonal anti-calf PARP-1 (clone C2-10; Trevigen, Gaithersburg, MD, USA; 1:2,000 dilution); mouse monoclonal anti-human Top1 (clone C-21 BD Biosciences; 1:500). Anti-rabbit and antimouse secondary antibodies (Sigma-Aldrich) were used at the appropriate dilutions. Signals were quantified using a Kodak densitometer (Rochester, NY, USA).

PARP activity assay. Cells $\left(5 \times 10^{6}\right)$ were lysed in $0.5 \mathrm{ml}$ of a buffer containing $0.1 \%$ Triton $\mathrm{X}-100,50 \mathrm{mM}$ Tris- $\mathrm{HCl} \mathrm{pH} 8.0$, $0.6 \mathrm{mM}$ EDTA, $14 \mathrm{mM} \beta$-mercaptoethanol, $10 \mathrm{mM} \mathrm{MgCl}_{2}$ and protease inhibitors. Proteins $(25 \mu \mathrm{g})$ were incubated with $2 \mu \mathrm{Ci}^{32} \mathrm{P}-\mathrm{NAD}^{+}$(GE Healthcare, Milan, Italy), $100 \mu \mathrm{M} \mathrm{NAD}^{+}$, $50 \mathrm{mM}$ Tris- $\mathrm{HCl}, 10 \mathrm{mM} \mathrm{MgCl}{ }_{2}, 14 \mathrm{mM} \beta$-mercaptoethanol, in the presence (maximally stimulated activity) or absence (basal activity) of $10 \mu \mathrm{g}$ nuclease-treated salmon testes DNA. After $15 \mathrm{~min}$ at $30^{\circ} \mathrm{C}$ the reaction was stopped adding ice-cold trichloroacetic acid $20 \%$ (v/v). The radioactivity associated with the acid-insoluble material, corresponding to poly (ADP-ribosyl)ated proteins, was counted on a liquid scintillation counter. PARP activity was evaluated as fmol of ${ }^{32} \mathrm{P}-\mathrm{NAD}+\mu \mathrm{g}$ of protein and data were presented as the ratio between maximally stimulated and basal activity. 
A

A

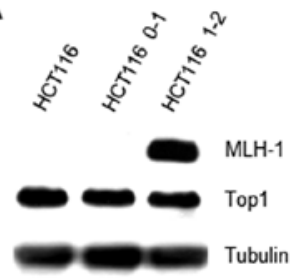

B
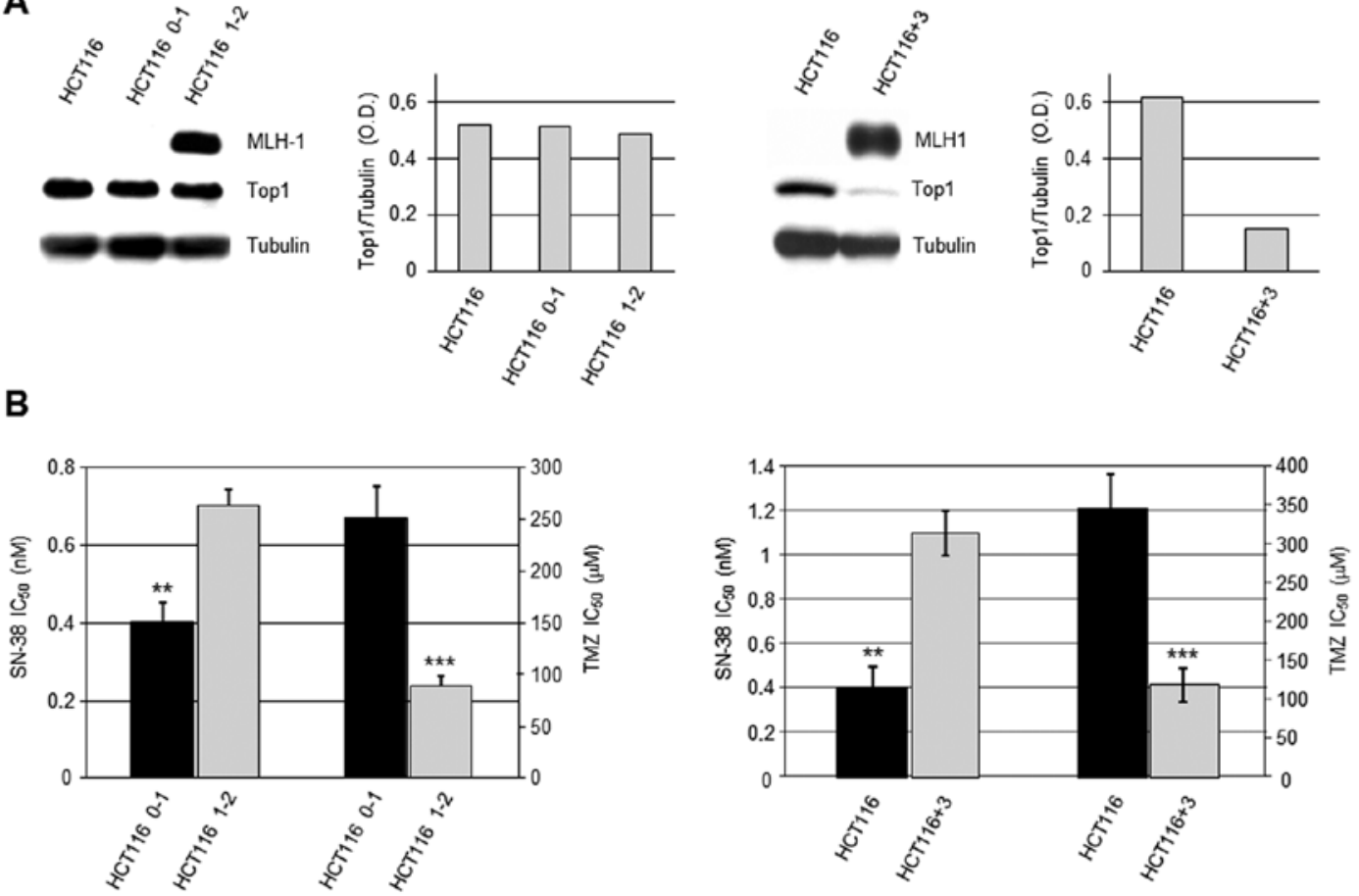

Figure 1. Analysis of MLH1 or Top1 expression and of sensitivity to SN-38 in HCT116 derived cell lines. (A) Analysis of MLH1 and Top1 expression. Cell lysates from parental HCT116 cells, HCT116 1-2 cells, transfected with MLH1 cDNA, and HCT116 0-1 cells, transfected with the empty control vector, or HCT116+3 cells, in which MLH1 had been introduced by chromosome transfer, were electrophoresed and analysed for the expression of MLH1, Top1 and tubulin. Histograms represent the ratios between the optical densities (OD) of Top1 and those of tubulin. The results are representative of one out of two experiments with similar results. (B) In vitro chemosensitivity. Tumor cell susceptibility to SN-38 or TMZ was assessed by colony-formation assay and the results were expressed as $\mathrm{IC}_{50}$. Only colonies comprising $>50$ cells were scored as survival colonies. Data are means $[ \pm$ standard deviation (SD)] from three independent experiments. The results of statistical analysis by Student's $t$-test of the differences in sensitivity are as follows: SN-38, HCT116 0-1 vs. HCT116 1-2, ${ }^{* *} \mathrm{P}=0.003$; TMZ, HCT116 0-1 vs. HCT116 1-2, ${ }^{* * *} \mathrm{P}<0.0001 ;$ SN-38, HCT116 vs. HCT116+3, ${ }^{* *} \mathrm{P}=0.002 ;$ TMZ, HT116 vs. HCT116+3, ${ }^{* * *} \mathrm{P}<0.0001$.

Immunofluorescence microscopy of $\gamma-H 2 A X$. Cells were grown on poly-L-lysine coated glass coverslips and treated with SN-38. After $24 \mathrm{~h}$, slides were washed twice with PBS and fixed in $4 \%$ (w/v) paraformaldehyde in PBS for $30 \mathrm{~min}$. Cells were permeabilised with $0.1 \%$ Triton X-100 in PBS for $2 \mathrm{~min}$, incubated in $2.5 \%$ goat serum-PBS for $20 \mathrm{~min}$ and with rabbit anti- $\gamma-\mathrm{H} 2 \mathrm{AX}$ polyclonal antibody for $2 \mathrm{~h}$ (Abcam, $1: 250$ in $2.5 \%$ goat serum-PBS). After washing in PBS, cells were incubated with goat anti-rabbit-Alexa 488 secondary antibody (Molecular Probes, Eugene, OR, USA; 1:2,000) for $1 \mathrm{~h}$. Slides were counterstained by vectashield antifade solution containing 4,6 diamidino-2-phenylindole (DAPI, Vector Laboratories, Burlingame, CA, USA) and examined with a fluorescent microscope (Nikon Eclipse, E600, Yokohama, Japan). Images were analysed with the Arkon FISH program (Nikon). For quantitative analysis, foci were counted by eye during the microscopic and imaging process using a x100 objective. Cells with $\geq 5$ foci were considered positive for $\gamma$-H2AX expression (28).

In vivo studies. The intramuscular transplantation procedure was performed as previously described (6). Tumor cells $\left(3 \times 10^{6}\right)$ were inoculated intramuscularly (i.m.) in male athymic CD-1 mice (nu/nu genotype, 6/group; Charles River, Calco, Milan, Italy). Xenograft growth was monitored by measuring tumor nodules with calliper every 2-3 days for 3 weeks and volumes were calculated according to the following formula: [(width ${ }^{2}$ x length]/2. Irinotecan (Campto ${ }^{\circledR}$, Aventis, Milan, Italy) was administere intraperitoneally (i.p) at $5 \mathrm{mg} / \mathrm{kg} / \mathrm{dx} 5 \mathrm{~d}$; treatment started when nodules reached $300 \mathrm{~mm}^{3}$. The animals were euthanized when their tumors reached a volume of $\sim 1500$ $\mathrm{mm}^{3}$. All procedures involving mice and care were performed in compliance with our institutional animal care guidelines and with international directives (directive 2010/63/EU of the European parliament and of the council; Guide for the Care and Use of Laboratory Animals, United States National Research Council, 2011). The study was approved by the ethics committee of the University of Rome 'Tor Vergata'.

\section{Results}

Analysis of MLH1 and Topl expression and of sensitivity to Topl inhibitor in HCT116 colon cancer cells transfected with the wild-type MLH1 cDNA or with chromosome 3. In order to evaluate the influence of MLH1 and Top1 or both in the susceptibility to the Top1 poison we initially performed immunoblot analysis of Top1 in the parental HCT116 cell line (devoid of MLH1 protein as a result of mutation in the MLH1 gene located on chromosome 3), in an HCT116 clone transfected with a control vector (HCT116 0-1) or with a vector expressing the wild-type MLH1 cDNA (HCT116 1-2) and in HCT116 cells in which MLH1 was introduced by transfer of chromosome 3 (HCT116+3). Our results indicated that HCT116, HCT116 0-1 and HCT116 1-2 expressed high and comparable levels of Top1 protein. On the other hand, HCT116+3 cells showed very low Top1 expression (Fig. 1A). Then we analysed the sensitivity 
A
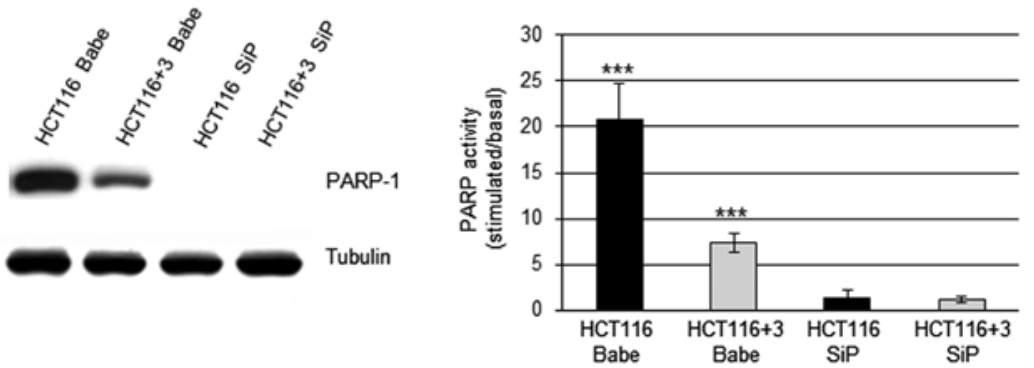

B

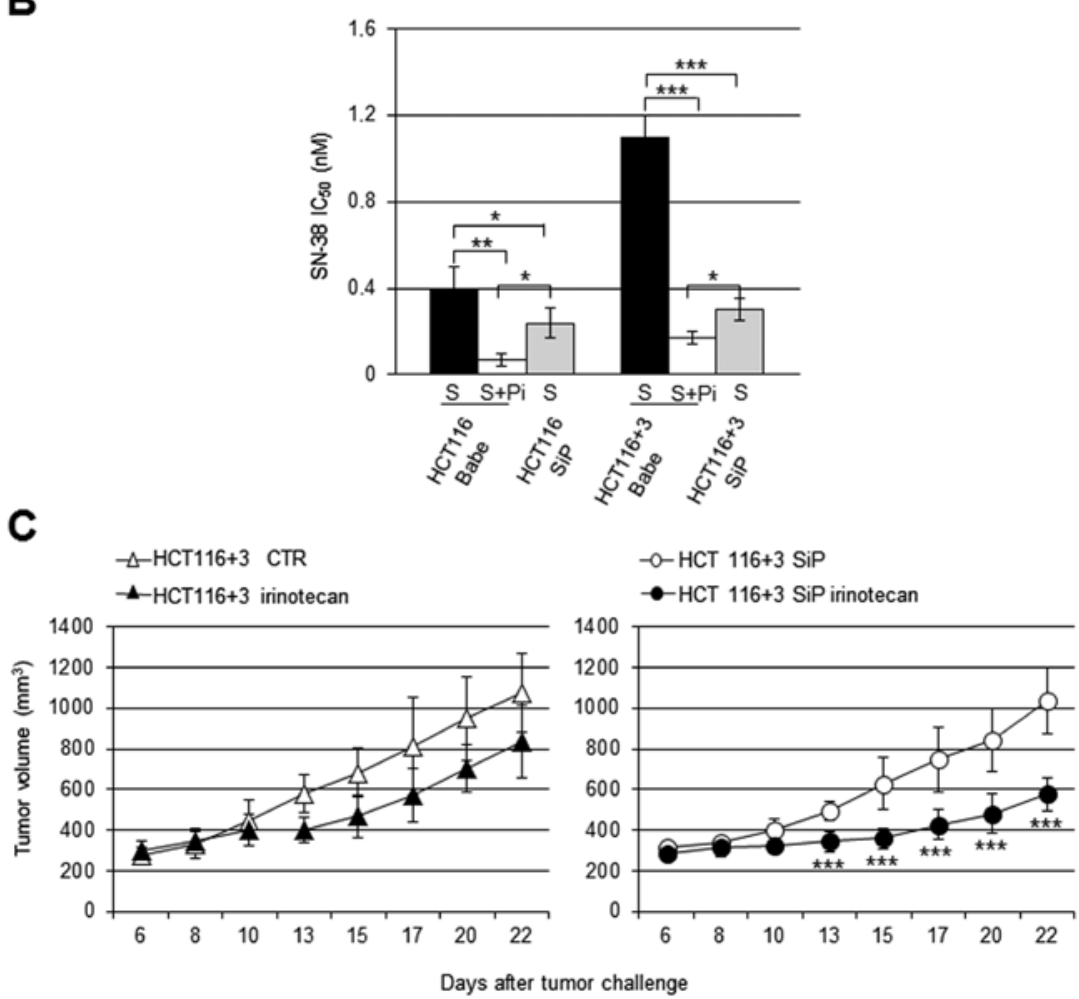

Figure 2. Sensitivity to SN-38 of HCT116 or HCT116+3 cells treated with a PARPi or stably silenced for PARP-1 expression. (A) Analysis of PARP-1 expression (left) and activity (right). Immunoblot analysis was performed in HCT116 or HCT116+3 clones transfected with the pBabe vector (HCT-116 Babe or HCT116+3 Babe) or with the pBabe and pBS-U6-SiP912 vectors (HCT-116 SiP or HCT116+3 SiP). Cellular extracts were electrophoresed and analysed for the expression of PARP-1 or tubulin. Total cellular PARP activity was measured in cell extracts, in the absence (basal activity) or in the presence of nuclease-treated DNA (maximally stimulated activity) and ${ }^{32} \mathrm{P}-\mathrm{NAD}^{+}$. The results were expressed as means $( \pm \mathrm{SD})$ of stimulated and basal activity ratios of three independent experiments. The results of statistical analysis by Student's $t$-test of the differences in PARP activity ratios are as follows: HCT116 Babe vs. HCT116+3 Babe, ${ }^{* * * *} \mathrm{P}<0.0001$; HCT116 Babe vs. HCT116 SiP, ${ }^{* * *} \mathrm{P}<0.0001$; HCT116+3 Babe vs. HCT116+3 SiP, ${ }^{* * *} \mathrm{P}<0.0001$; HCT116 SiP vs. HCT116+3 SiP; NS, not significant. (B) Comparison of HCT116 or HCT116+3 sensitivity to SN-38, as single agent or in combination with a PARPi, with that of PARP-1 silenced HCT116 or HCT116+3 cells. Control HCT116 or HCT116+3 (Babe) or PARP-1 silenced (SiP) cells were exposed to graded concentrations of SN-38 (S) and analysed by colony-formation assay. In the case of Babe cells SN-38 was also combined with the PARPi GPI 15427 (Pi), at a concentration $(0.3 \mu \mathrm{M})$ capable of significantly inhibiting cellular PARP activity (70-75\% inhibition of the synthesis of ADP-ribose polymers) and devoid of a significant growth inhibitory effect (<15\%). The results were expressed as $\mathrm{IC}_{50}$. Data are means $( \pm \mathrm{SD})$ from three independent experiments. The results of statistical analysis by Student's $t$-test of the differences in sensitivity are as follows: HCT116 Babe treated with SN-38 vs. HCT116 Babe treated with SN-38+PARPi, ${ }^{* *} \mathrm{P}=0.004 ; \mathrm{HCT} 116 \mathrm{Babe}$ treated with SN-38 vs. HCT116 SiP treated with SN-38, ${ }^{*} \mathrm{P}=0.04$; HCT116 Babe treated with SN-38+PARPi vs. HCT116 SiP treated with SN-38, "P=0.01; HCT116+3 Babe treated with SN-38 vs. HCT116+3 Babe treated with SN-38+PARPi, ${ }^{* * *} \mathrm{P}<0.0001$; HCT116+3 Babe treated with SN-38 vs. HCT116+3 SiP treated with SN-38, ${ }^{* * *} \mathrm{P}<0.0001$; HCT116+3 Babe treated with SN-38+PARPi vs. HCT116+3 SiP treated with SN-38, ${ }^{*} \mathrm{P}=0.02 ;$ HCT116 SiP treated with SN-38 vs. HCT116+3 SiP treated with SN-38; NS, not significant. (C) Analysis of in vivo chemosensitivity of HCT116+3 Babe and SiP cells in mice treated with irinotecan. Mice were inoculated i.m. with Babe $(\mathrm{n}=6)$ or $\mathrm{SiP}(\mathrm{n}=6) \mathrm{HCT} 116+3$ cells and treated with irinotecan (as indicated in Materials and methods). Statistical analysis by Student's $t$-test indicated that the growth of SiP grafts is significantly inhibited by irinotecan compared with untreated control grafts $\left({ }^{* * * *} \mathrm{P}<0.001 \mathrm{from}\right.$ day 13 onward); differences between the volumes of tumor nodules of untreated or drug treated mice injected with HCT116+3, NS, not significant.

of MLH1-deficient and -proficient cells to SN-38, the active metabolite of irinotecan and to the methylating agent TMZ, as a control for MLH1 functional proficiency. In fact, MLH1 is required for the processing and cytotoxicity of $\mathrm{O}^{6} \mathrm{MethylG} / \mathrm{T}$ mispairs generated by TMZ. The results, expressed as $\mathrm{IC}_{50}$, indicated that the MLH1-deficient HCT116 0-1 and HCT116 cells were more sensitive to SN-38 as compared to MLH1proficient HCT116 1-2 and HCT116+3 cells (Fig. 1B). The lower constitutive expression of Top1 might contribute to the higher SN-38 resistance of HCT116+3 cells compared with HCT116 1-2 cells (Fig. 1A). In HCT116 and HCTT116+3 cells the breast cancer resistance protein (BCRP), an ATP binding 
A

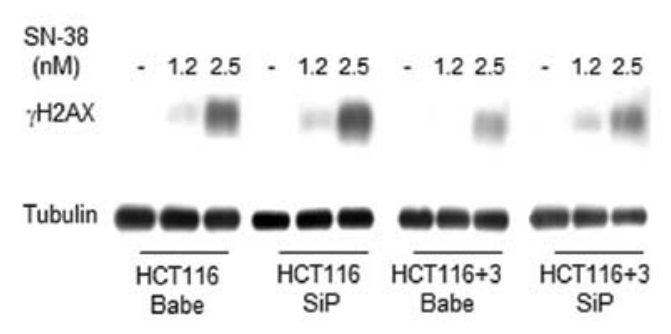

B
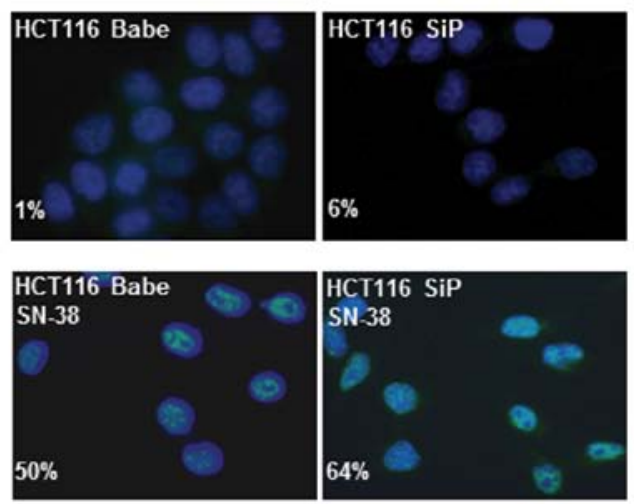

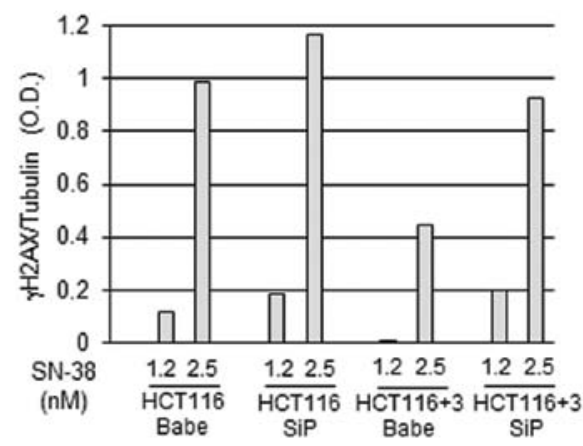

$\mathrm{Bab}$
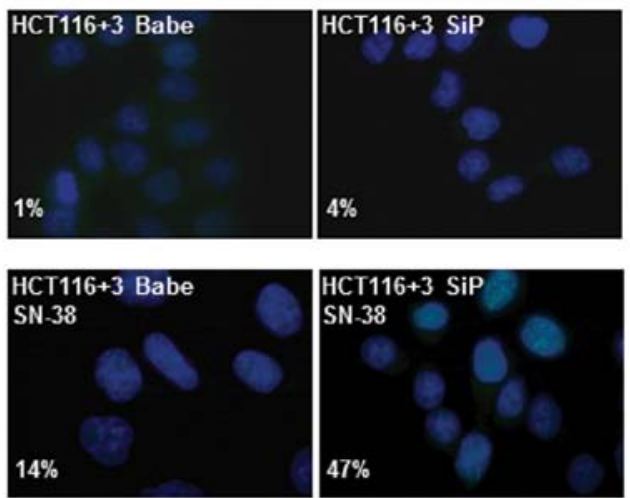

Figure 3. Analysis of DNA damage induced by SN-38 in HCT116 or HCT116+3 cells control or stably silenced for PARP-1 expression. (A) Immunoblot analysis of $\gamma$-H2AX expression in SN-38 treated cells. Cells were treated with SN-38 (1.2 and $2.5 \mathrm{nM}$ ) for $24 \mathrm{~h}$ and analysed for the expression of $\gamma$-H2AX or tubulin. Histograms represent the ratios between the OD of $\gamma-\mathrm{H} 2 \mathrm{AX}$ in SN-38 treated groups and tubulin. The results are representative of one out of two experiments with similar results. (B) Immunofluorescence analysis of $\gamma$-H2AX foci (green) in untreated or SN-38 treated cells. Nuclei were stained with DAPI (blue). The percentage of cells with $\geq 5 \gamma$-H2AX foci of one representative out of two experiments is presented (50 cells counted for each experiment).

cassette transporter, which is regarded as an important determinant of resistance to camptothecins, was, instead, equally expressed (6). On the contrary, MLH1 proficiency conferred sensitivity to TMZ to a similar extent in both cellular models (Fig. 1B).

Lack of PARP-1 activity increases the sensitivity to irinotecan in MLH1-proficient and-deficient HCT116 cells. We then evaluated the influence on tumor sensitivity to SN-38 of downregulation of PARP function by PARP-1 gene silencing and by pharmacological inhibition of poly(ADP-ribose) synthesis using PARPi. To this end HCT116 and HCT116+3 were stably silenced for PARP-1 expression (SiP clones) by transfection of a siRNA vector targeting specific sequences of PARP-1, which is responsible for $>90 \%$ of the cellular poly(ADP-ribosyl) ating activity. Control clones were obtained by transfection of the pBabe vector only (Babe clones). Immunoblot analysis and PARP activity assay (Fig. 2A) revealed lack of PARP-1 expression and negligible synthesis of poly(ADP-ribose) in $\mathrm{SiP}$ clones. Moreover, HCT116 Babe cells showed significantly higher levels of PARP-1 expression and activity compared with HCT116+3 Babe cells.

Then, control Babe clones were tested for their susceptibility to the anti-proliferative effects of SN-38 in combination with a PARPi and the results compared to those obtained in their SiP counterparts. Pharmacological inhibition of PARP activity by GPI 15427 significantly increased sensitivity to SN-38 both in MLH1-deficient and in MLH1-proficient cells, as indicated by the $\mathrm{IC}_{50}$ values of SN-38 in the presence of PARPi that were $\sim 6$-fold lower than those of SN-38 used as single agent (Fig. 2B). PARP-1 silenced clones were 2 -4-fold more susceptible to the Top1 poison than control clones (Fig. 2B). The enhancing effect mediated by PARPi or PARP-1 gene silencing was more pronounced in the less sensitive HCT116+3 cells (Fig. 2B). Noteworthy, in vivo studies showed that lack of PARP-1 expression in HCT116+3 SiP cells resulted in a higher tumor growth inhibition induced by irinotecan compared with HCT116+3 Babe cells (Fig. 2C). Analysis of the doubling times, evaluated on the basis of the growth kinetics of each clone, indicated that SiP clones possessed doubling times in the same range of those observed in PARP-1 proficient clones (22-26 h). Therefore, the distinct chemosensitivity profiles of Babe and SiP clones did not depend on different proliferation rates.

According to the results of colony formation assay, immunoblot analysis of histone $\mathrm{H} 2 \mathrm{AX}$ phosphorylation $(\gamma-\mathrm{H} 2 \mathrm{AX})$, as an indicator of DSB, showed that treatment with SN-38 induced histone phosphorylation at higher level in HCT116 Babe than in HCT116+3 Babe cells and in PARP-1 silenced clones with respect to their PARP-1 proficient controls (Fig. 3A). Immunofluorescence analysis of $\gamma-\mathrm{H} 2 \mathrm{AX}$ foci in cells treated with $\mathrm{SN}-38$ confirmed the results of immunon- 
A
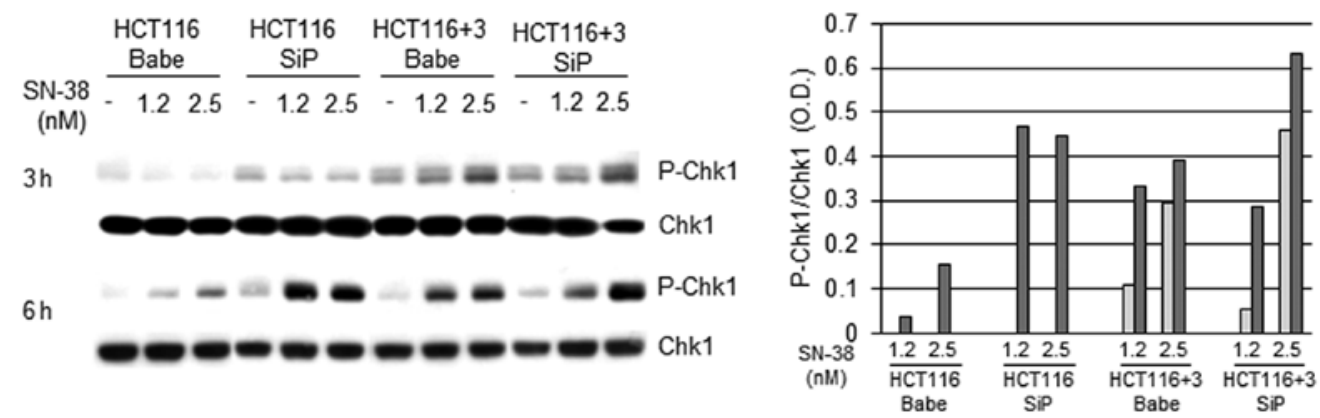

B

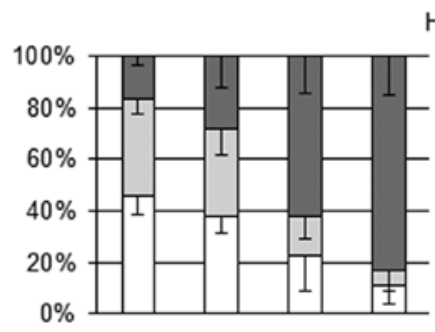

HCT116+3 Babe

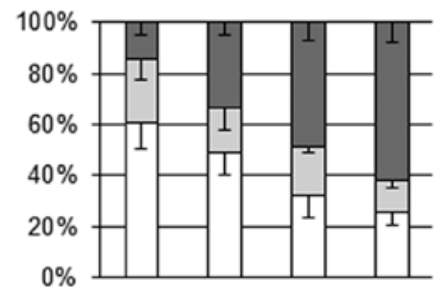

HCT116+3 SiP
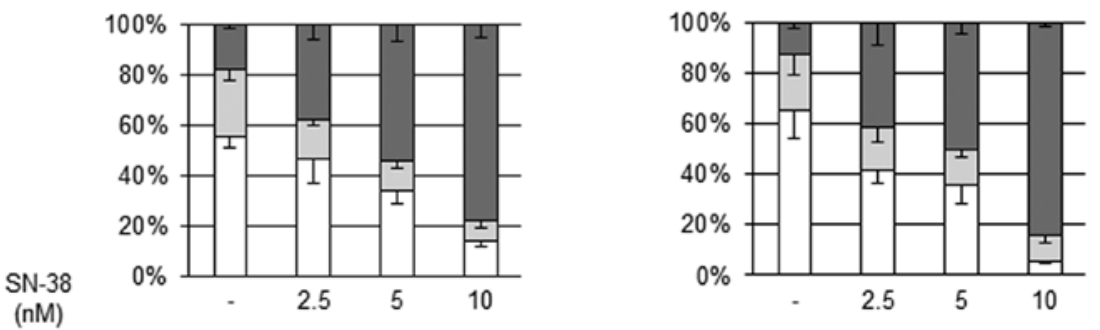

Figure 4. Analysis of Chk1 phosphorylation and cell cycle in Babe and SiP HCT116 and HCT116+3 cells treated with SN-38. (A) Immunoblot analysis of Chk1 phosphorylation. HCT116 and HCT116+3 Babe or SiP cells were treated with SN-38 (1.2 and $2.5 \mathrm{nM})$ for 3 and 6 h; cell lysates were electrophoresed and analysed for the expression of phosphorylated Chk1 (P-Chk1) or Chk1. Histograms represent the ratios between the OD of phosphorylated Chk1 (after subtraction of OD values of untreated controls) and $\mathrm{Chk} 1 \mathrm{in} \mathrm{SN}-38$ treated groups ( $3 \mathrm{~h}$, light grey columns; $6 \mathrm{~h}$, dark grey columns). The results are representative of one out of two experiments with comparable results.(B) Cell cycle analysis. HCT116+3 Babe or SiP cells were treated with SN-38 (2.5-10 nM) and analysed by flow cytometry. The results are indicated as percentages of cells in the different phases of cell cycle at $24 \mathrm{~h}$ (left) and $72 \mathrm{~h}$ (right) after treatment and are the means (- SD) from three independent experiments. G1 phase, white columns; S phase, light grey columns; M phase, dark grey columns.

oblot analysis of $\gamma$-H2AX expression (Fig. 3B). The data also showed that the increment of $\gamma-\mathrm{H} 2 \mathrm{AX}$ expression and foci formation resulting from the comparison of $\mathrm{HCT} 116+3 \mathrm{SiP}$ with HCT116+3 Babe cells was higher than that deriving from HCT116 SiP and HCT116 Babe comparison (Fig. 3).

Since MLH1 is known to be involved in DNA damageinduced checkpoint, signalling the control of $\mathrm{G} 2 / \mathrm{M}$ arrest by methylating agents like TMZ through phosphorylation of Chk1 (25-27), we have investigated whether treatment with SN-38 might induce different kinetics of induction of Chk1 phosphorylation and cell cycle perturbations depending on the presence of MLH1 and/or PARP-1. The results of immunoblot analysis of Chk1 phosphorylation revealed that SN-38 treatment induced an earlier Chk1 phosphorylation ( $3 \mathrm{~h})$ in MLH1-proficient cells than in MLH1-deficient cells (Fig. 4A). At a later time-point ( $6 \mathrm{~h}$ ) the highest level of Chk1 phosphorylation was detected in cells silenced for PARP-1, regardless of MLH1 expression (Fig. 4A). Cell treatment with SN-38 did not result in Chk2 phosphorylation (data not shown). Cell cycle analysis at $24 \mathrm{~h}$ after treatment showed a dose-dependent cell accumulation at the G2/M phase (Fig. 4B for HCT116+3 Babe and Sip cells; data not shown for HCT116 Babe and Sip cells). At $72 \mathrm{~h}$ after treatment the percentage of cells in the $\mathrm{G} 2 / \mathrm{M}$ phase decreased in both Babe cell lines, whereas both PARP-1 silenced cells underwent G2/M arrest (Fig. 4B for HCT116+3 Babe and SiP cells; data not shown for HCT116 Babe and SiP cells).

In HCT116 MLH1-deficient Babe and SiP clones treatment with SN-38 induced a dose-dependent and marked increase of p53 phosphorylation and of the percentage of apoptotic cells; this effect was more pronounced in PARP-1 silenced cells (Fig. 5). On the other hand, in HCT116+3 Babe and SiP cells only marginal level of p53 phosphorylation and apoptosis were detected (Fig. 5). Treatment of HCT116+3 Babe and $\mathrm{SiP}$ cells with a non-toxic concentration of the Chk1 inhibitor UCN-01 reduced the G2/M cell accumulation (data not shown) favouring apoptosis induction by $\mathrm{SN}-38$ in both cell lines (Fig. 5B). 
A
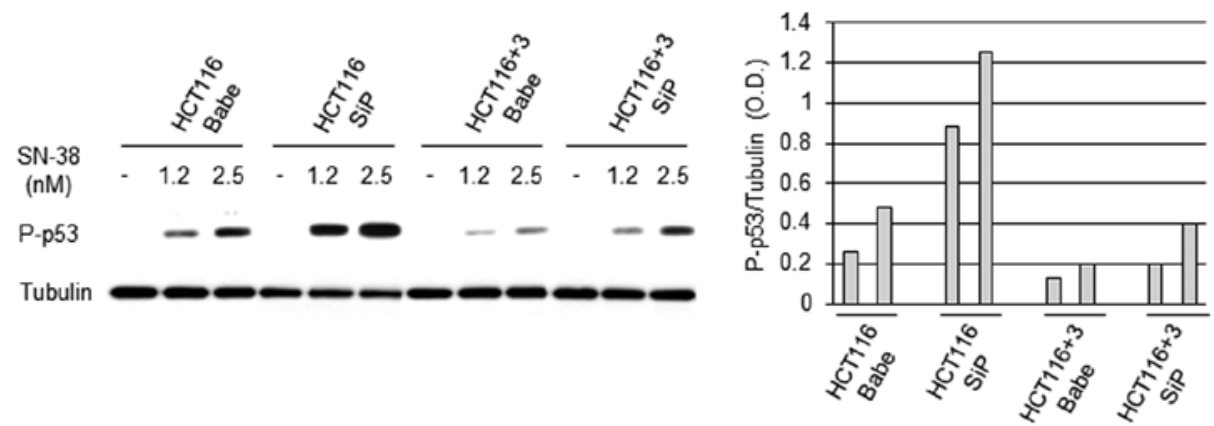

B
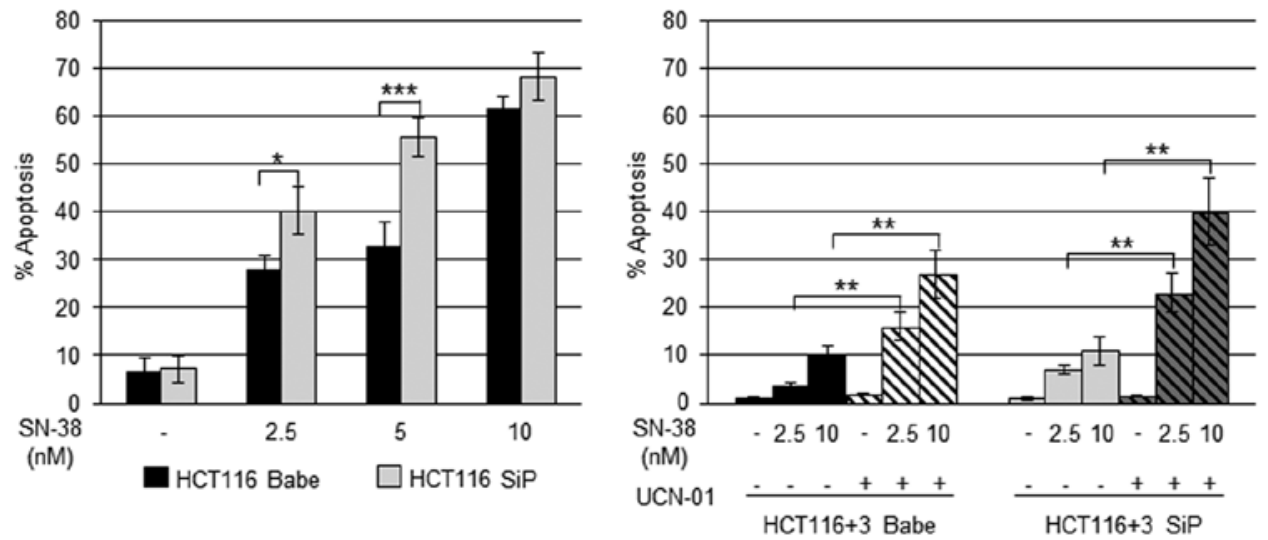

Figure 5. Analysis of p53 activation (A) and apoptosis induction (B) in MLH1-deficient HCT116 Babe and SiP cells treated with SN-38. (A) HCT116 and $\mathrm{HCT} 116+3$ Babe or SiP cells were treated with the indicated concentrations of SN-38 for $6 \mathrm{~h}$; cell lysates were electrophoresed and analysed for the expression of phosphorylated p53 (P-p53) or tubulin. Histograms represent the ratios between the OD of phospho-p53 in SN-38 treated groups and tubulin. The results are representative of one out of two experiments with similar results. (B) Apoptosis was analysed by flow cytometry in HCT116 and HCT116+3 Babe or SiP cells

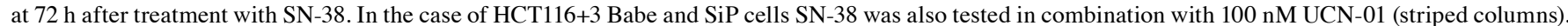
Histograms represent the mean percentage values $( \pm \mathrm{SD})$ of apoptotic cells from three independent experiments. The results of statistical analysis by Student's $t$-test of the differences in sensitivity to apoptosis induction are as follows: left histogram, $2.5 \mathrm{nM} \mathrm{SN}-38$ HCT116 Babe vs. HCT116 SiP, ${ }^{*} \mathrm{P}=0.02 ; 5 \mathrm{nM}$ SN-38, HCT116 Babe vs. HCT116 SiP, ${ }^{* * *} \mathrm{P}=0.004 ; 10 \mathrm{nM}$ SN-38 HCT116 Babe vs. HCT116 SiP; NS, not significant; right histogram, HCT116+3 Babe, $2.5 \mathrm{nM}$ SN-38 vs. $2.5 \mathrm{nM} \mathrm{SN}-38+\mathrm{UCN}-01,{ }^{* *} \mathrm{P}=0.002 ; 10 \mathrm{nM} \mathrm{SN}-38$ vs. $10 \mathrm{nM} \mathrm{SN}-38+\mathrm{UCN}-01,{ }^{* *} \mathrm{P}=0.005 ;$ HCT116+3 SiP, $2.5 \mathrm{nM}$ SN-38 vs. $2.5 \mathrm{nM}$ SN-38+UCN-01, ${ }^{* *} \mathrm{P}=0.002 ; 10 \mathrm{nM} \mathrm{SN}-38$ vs. $10 \mathrm{nM} \mathrm{SN}-38+\mathrm{UCN}-01,{ }^{* *} \mathrm{P}=0.003$.

\section{Discussion}

Germline mutations of MMR genes or epigenetic inactivation of MLH1 may be present in CRC and influence the clinical behavior $(28,29)$. While the lack of function of MLH1 and the presence of microsatellite instability have been associated with lower response to 5-FU (30), the predictive impact of the MLH1 functional status for irinotecan, as single agent or in combination with PARPi, needs to be clarified. Using a panel of human colon cancer cell lines derived from HCT116 and characterized by different patterns of MLH1, Top1 and PARP-1 expression, we investigated the influence of these proteins on colon cancer cell response to irinotecan, as single agent or in combination with PARPi. To study the impact of MLH1 expression on colon cancer cell chemosensitivity we used cell lines in which the defective gene product was restored by chromosome transfer or by transfection of the corresponding wild-type cDNA. The results indicated that MLH1-proficiency and low Top1 expression resulted in reduced sensitivity to irinotecan, but pharmacological inhibition of PARP activity or silencing of PARP-1 gene increased the susceptibility of colon cancer cells to the Top1 poison. Interestingly, cells with lower levels of PARP-1 protein could still be sensitized to irinotecan by PARPi.

DNA damage consequent to Top1 poisoning derives from stabilization of the cleavable complex formed by Top1 and DNA, with formation of a transient single strand DNA nick. When the stabilized drug-DNA-Top1 complex collides with replication or transcription machineries, it leads to replication fork stalling and eventually DSB. Before DSB generation, the repair of DNA damage induced by Top1 inhibitors includes proteasomal degradation of Top1, hydrolysis of the phosphodiester bond, between the 3'-end of DNA and a tyrosine residue of Top1, by the tyrosyl DNA phosphodiesterase-1 and removal of Top1-cleavable complex by the endonucleases XPF-ERCC1 (2). In MLH1-proficient cells SN-38 induced a lower level of DNA damage with respect to MLH1-deficient cells, as evidenced by the weak induction of $\gamma-\mathrm{H} 2 \mathrm{AX}$ and p53 phosphorylation. The presence of MLH1 contributed to induce prompt Chk1 phosphorylation, restoring G2/M cell cycle checkpoint and repair of DNA damage. On the contrary, in the absence of MLH1, HCT116 cells showed minor Chk1 
phosphorylation and underwent apoptosis. Actually, inhibition of Chk1 by UCN-01 decreased G2/M cell accumulation induced by the Top1 poison in MLH1-proficient cells and triggered apoptosis. Treatment with SN-38 did not result in Chk2 phosphorylation (data not shown) likely for the presence of mutations in the Mre11 gene. The consequent lack of Mre11 protein (31) and very low expression of RAD50 cause destabilization of the MRN complex, which is required for Chk2 phosphorylation by ATM in response to DSB (32).

Low levels of Top1 protein are known to confer a moderate level of resistance to camptothecins due to the formation of a small amount of DNA-Top1 cleavable complexes (2). Since HCT116+3 cells expressed lower level of Top1 protein with respect to HCT116 cells, this might have contributed to the poorer response of HCT116+3 cells to irinotecan. In fact, the sensitivity to SN-38 of HCT116 1-2 cells transfected with the MLH1 cDNA differed to a lesser extent from that of HCT116 control cells, which showed comparable Top1 expression. Our data indicated that when a defective gene product is replaced by chromosome transfer the expression of other proteins involved in tumor drug response may change and influence tumor chemosensitivity.

Pharmacological inhibition or stable gene silencing of PARP-1 increased sensitivity to SN-38 both in MLH1proficient and -deficient cells. Notably, the enhancing effect deriving from abrogation of PARP-1 function was particularly evident in the HCT116+3 derived line and translated into an increased in vivo response of $\mathrm{HCT} 116+3 \mathrm{SiP}$ to irinotecan. Tumor chemosensitization mediated by lack of function of PARP-1 is likely due to the decrease of DNA repair mediated by the BER system. PARP-1 contributes to the repair of Top1 mediated damage promoting the recruitment of XRRC1 and tyrosyl DNA phosphodiesterase-1 with removal of Top1 from DNA (5). These results are consistent with the reported higher toxicity of Top1 poisons in cervical cancer or in melanoma cells stably silenced for PARP-1 $(33,34)$. On the other hand, the transient silencing of PARP-1 in an ovarian cancer cell line did not result in chemosensitisation to Top1 poisons (35). During the preparation of this study, a report was published indicating that the PARPi veliparib synergized with irinotecan in vitro in HCT116 cells (36). In our study we showed, for the first time, that the chemosensitising effect induced by pharmacological inhibition of PARP or by PARP-1 gene silencing can be achieved even in the presence of low Topl levels and in the case of both MLH1-proficient and MLH1-deficient tumors.

In PARP-1 silenced cells devoid also of MLH1 expression SN-38 induced more DNA damage than in MLH1-proficient SiP cells, causing a remarkable p53 phosphorylation and apoptosis induction. In MLH1-deficient SiP cells the Top1 poison also induced a delayed (compared with MLH1-proficient SiP) but robust Chk1 phosphorylation, suggesting that MLH1 might influence the kinetics of induction rather than the extent of Chk1 post-translational modifications.

Interestingly, the low levels of PARP-1 expression in HCT116+3 cells did not hamper the chemosensitising effect mediated by the PARPi GPI 15427 in combination with $\mathrm{SN}-38$. These results are different from those obtained in MEF lines derived from PARP-1/- mice that could not be sensitized to topotecan by the PARPi veliparib (35). However, in MEF cells PARP-1 was totally lacking, whereas in HCT116+3 cells
PARP-1, even though expressed at lower levels than in HCT116 cells, was sufficient to allow the chemosensitising effect by GPI 15427.

In conclusion, these data indicated that MLH1, together with low levels of Top1, contributes to colon cancer resistance to Top1 poisons. Remarkably, inhibition of PARP-1 function always increases the antitumor activity of irinotecan even in the presence of low PARP-1 expression.

\section{Acknowledgements}

This study was supported by a grant from the Italian Ministry of Education and Research, 'Programmi di ricerca scientifica di Rilevante Interesse Nazionale' (PRIN) project to L.T. A.M. is recipient of a fellowship from 'Regione Lazio-Filas'.

\section{References}

1. Davies JM and Goldberg RM: Treatment of metastatic colorectal cancer. Semin Oncol 38: 552-560, 2011.

2. Alagoz M, Gilbert DC, El-Khamisy S and Chalmers AJ: DNA repair and resistance to topoisomerase I inhibitors: mechanisms, biomarkers and therapeutic targets. Curr Med Chem 19: 3874-3885, 2012.

3. Miknyoczki SJ, Jones-Bolin S, Pritchard S, Hunter K, Zhao H, Wan W, Ator M, et al: Chemopotentiation of temozolomide, irinotecan and cisplatin activity by CEP-6800, a poly(ADPribose) polymerase inhibitor. Mol Cancer Ther 2: 371-382, 2003.

4. Calabrese CR, Almassy R, Barton S, Batey MA, Calvert AH, Canan-Koch S, Durkacz BW, et al: Anticancer chemosensitization and radiosensitization by the novel poly(ADP-ribose) polymerase-1 inhibitor AG14361. J Natl Cancer Inst 96: 56-67, 2004.

5. Smith LM, Willmore E, Austin CA and Curtin NJ: The novel poly(ADP-Ribose) polymerase inhibitor, AG14361, sensitizes cells to topoisomerase I poisons by increasing the persistence of DNA strand breaks. Clin Cancer Res 11: 8449-8457, 2005.

6. Tentori L, Leonetti C, Scarsella M, Muzi A, Mazzon E, Vergati M, Forini O, et al: Inhibition of poly(ADP-ribose) polymerase prevents irinotecan-induced intestinal damage and enhances irinotecan/temozolomide efficacy against colon carcinoma. FASEB J 20: 1709-1711, 2006.

7. Daniel RA, Rozanska AL, Thomas HD, Mulligan EA, Drew Y, Castelbuono DJ, Hostomsky Z, et al: Inhibition of poly(ADPribose) polymerase-1 enhances temozolomide and topotecan activity against childhood neuroblastoma. Clin Cancer Res 15: 1241-1249, 2009

8. Schreiber V, Dantzer F, Ame JC and de Murcia G: Poly(ADPribose): novel functions for an old molecule. Nat Rev Mol Cell Biol 7: 517-528, 2006.

9. Malanga M and Althaus FR: Poly(ADP-ribose) reactivates stalled DNA topoisomerase I, induces DNA strand break resealing. J Biol Chem 279: 5244-5248, 2004.

10. Leonetti C, Biroccio A, Graziani G and Tentori L: Targeted therapy for brain tumours: role of PARP inhibitors. Curr Cancer Drug Targets 12: 218-236, 2012.

11. Martin SA, Lord CJ and Ashworth A: Therapeutic targeting of the DNA mismatch repair pathway. Clin Cancer Res 16: 5107-5113, 2010.

12. D'Atri S, Tentori L, Lacal PM, Graziani G, Pagani E, Benincasa E, Zambruno G, et al: Involvement of the mismatch repair system in temozolomide-induced apoptosis. Mol Pharmacol 54: 334-341, 1998.

13. Tentori L and Graziani G: Recent approaches to improve the antitumor efficacy of temozolomide. Curr Med Chem 16: 245-257, 2009.

14. Fedier A, Schwarz VA, Walt H, Carpini RD, Haller U and Fink D: Resistance to topoisomerase poisons due to loss of DNA mismatch repair. Int J Cancer 93: 571-576, 2001.

15. Jacob S, Aguado M, Fallik D and Praz F: The role of the DNA mismatch repair system in the cytotoxicity of the topoisomerase inhibitors camptothecin and etoposide to human colorectal cancer cells. Cancer Res 61: 6555-6562, 2001. 
16. Magrini R, Bhonde MR, Hanski ML, Notter M, Scherübl H, Boland CR, Zeitz M and Hanski C: Cellular effects of CPT-11 on colon carcinoma cells: dependence on p53 and hMLH1 status. Int J Cancer 101: 23-31, 2002.

17. Fallik D, Borrini F, Boige V, Viguier J, Jacob S, Miquel C, Sabourin JC, et al: Microsatellite instability is a predictive factor of the tumor response to irinotecan in patients with advanced colorectal cancer. Cancer Res 63: 5738-5744, 2003.

18. Papouli E, Cejka P and Jiricny J: Dependence of the cytotoxicity of DNA-damaging agents on the mismatch repair status of human cells. Cancer Res 64: 3391-3394, 2004.

19. Bhonde MR, Hanski ML, Stehr J, Jebautzke B, Peiró-Jordán R, Fechner H, Yokoyama KK, et al: Mismatch repair system decreases cell survival by stabilizing the tetraploid G1 arrest in response to SN-38. Int J Cancer 126: 2813-2825, 2010.

20. Martin LP, Hamilton TC and Schilder RJ: Platinum resistance: the role of DNA repair pathways. Clin Cancer Res 14: 1291-1295, 2008.

21. Papadopoulos N, Nicolaides NC, Wei YF, Ruben SM, Carter KC, Rosen CA, Haseltine WA, et al: Mutation of a mutL homolog in hereditary colon cancer. Science 263: 1625-1629, 1994.

22. Koi M, Umar A, Chauhan DP, Cherian SP, Carethers JM, Kunkel TA and Boland CR: Human chromosome 3 corrects mismatch repair deficiency and microsatellite instability and reduces $\mathrm{N}$-methyl-N'-nitro-N-nitrosoguanidine tolerance in colon tumor cells with homozygous hMLH1 mutation. Cancer Res 54: 4308-4312, 1994.

23. Vernole P, Muzi A, Volpi A, Terrinoni A, Dorio AS, Tentori L, Shah GM and Graziani G: Common fragile sites in colon cancer cell lines: role of mismatch repair, RAD51 and poly(ADP-ribose) polymerase-1. Mutat Res 712: 40-48, 2011.

24. Tentori L, Leonetti C, Scarsella M, D'Amati G, Vergati M, Portarena I, Xu W, et al: Systemic administration of GPI 15427, a novel poly(ADP-ribose) polymerase-1 inhibitor, increases the antitumor activity of temozolomide against intracranial melanoma, glioma, lymphoma. Clin Cancer Res 9: 5370-5379, 2003.

25. Yamane K, Taylor K and Kinsella TJ: Mismatch repair-mediated G2/M arrest by 6-thioguanine involves the ATR-Chk1 pathway. Biochem Biophys Res Commun 318: 297-302, 2004.

26. Yoshioka K, Yoshioka Y and Hsieh P: ATR kinase activation mediated by MutSalpha and MutLalpha in response to cytotoxic O6-methylguanine adducts. Mol Cell 22: 501-510, 2006.
27. Adamson AW, Beardsley DI, Kim WJ, Gao Y, Baskaran R and Brown KD: Methylator-induced, mismatch repair-dependent G2 arrest is activated through Chk1 and Chk2. Mol Biol Cell 16: 1513-1526, 2005

28. Marra G and Jiricny J: DNA mismatch repair and colon cancer. Adv Exp Med Biol 570: 85-123, 2005.

29. Damia G and D'Incalci M: Genetic instability influences drug response in cancer cells. Curr Drug Targets 11: 1317-1324, 2010.

30. Sinicrope FA, Foster NR, Thibodeau SN, Marsoni S, Monges G, Labianca R, Kim GP, et al: DNA mismatch repair status and colon cancer recurrence and survival in clinical trials of 5-fluorouracilbased adjuvant therapy. J Natl Cancer Inst 103: 863-875, 2011.

31. Garner KM and Eastman A: Variations in Mre11/Rad50/Nbs1 status and DNA damage-induced S-phase arrest in the cell lines of the NCI60 panel. BMC Cancer 206: 1-13, 2011.

32. Takemura H, Rao VA, Sordet O, Furuta T, Miao ZH, Meng L, Zhang $\mathrm{H}$ and Pommier Y: Defective Mre11-dependent activation of Chk2 by ataxia telangiectasia mutated in colorectal carcinoma cells in response to replication-dependent DNA double strand breaks. J Biol Chem 281: 30814-30823, 2006.

33. Tentori L, Muzi A, Dorio AS, Scarsella M, Leonetti C, Shah GM, $\mathrm{Xu}$ W, et al: Pharmacological inhibition of poly(ADP-ribose) polymerase (PARP) activity in PARP-1 silenced tumour cells increases chemosensitivity to temozolomide and to a N3-adenine selective methylating agent. Curr Cancer Drug Targets 10: 368-383, 2010.

34. D'Onofrio G, Tramontano F, Dorio AS, Muzi A, Maselli V, Fulgione D, Graziani G, et al: Poly(ADP-ribose) polymerase signaling of topoisomerase 1-dependent DNA damage in carcinoma cells. Biochem Pharmacol 81: 194-202, 2011.

35. Patel AG, Flatten KS, Schneider PA, Dai NT, McDonald JS, Poirier GG and Kaufmann SH: Enhanced killing of cancer cells by poly(ADP-ribose) polymerase inhibitors and topoisomerase I inhibitors reflects poisoning of both enzymes. J Biol Chem 287: 4198-4210, 2012.

36. Davidson D, Wang Y, Aloyz R and Panasci L: The PARP inhibitor ABT-888 synergizes irinotecan treatment of colon cancer cell lines. Invest New Drugs 31: 461-468, 2013. 